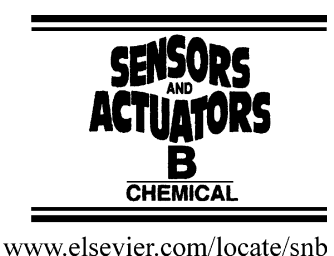

\title{
Microparticle photometry in a CMOS microsystem combining magnetic actuation and in situ optical detection
}

\author{
U. Lehmann ${ }^{\mathrm{a}, *}$, M. Sergio ${ }^{\mathrm{b}}$, S. Pietrocola ${ }^{\mathrm{a}}$, E. Dupont ${ }^{\mathrm{a}}$, \\ C. Niclass ${ }^{\text {b }}$, M.A.M. Gijs ${ }^{\text {a }}$, E. Charbon ${ }^{\mathrm{b}}$ \\ ${ }^{a}$ Institute of Microelectronics and Microsystems, Microsystems Laboratory 2, Ecole Polytechnique Fédérale de Lausanne (EPFL), Lausanne, Switzerland \\ ${ }^{\mathrm{b}}$ School of Computer and Communication Sciences, Ecole Polytechnique Fédérale de Lausanne (EPFL), Lausanne, Switzerland
}

\begin{abstract}
We present a hybrid CMOS-based microfluidic system that combines magnetic actuation of microparticles with in situ optical detection using single photon avalanche diodes (SPADs). The decoupling of the principles used for actuation and sensing permits a high sensitivity with respect to detection and particle handling. Single magnetic microparticles are transported within a glass micro-capillary positioned over an array of actuation coils and are detected upon passage over a SPAD, where they block incident light and thus lower the photon count. Use of the photometry method allows the determination of the particle size, which, in combination with a simultaneous measurement of the particle velocity, enables us to estimate further particle properties, such as their magnetization. We present the successful manipulation, detection and evaluation of magnetic particles with diameters ranging from 1 to $30 \mu \mathrm{m}$.
\end{abstract}

(C) 2007 Elsevier B.V. All rights reserved.

Keywords: Microfluidics; CMOS; Microparticles; Optical detection; Magnetism; SPAD; Photometry

\section{Introduction}

Magnetic microparticles and their application in bioanalytical microfluidic systems have been steadily gaining interest in recent years [1-3]. This progress is largely caused by the advantage of generating comparatively large and long-range magnetic forces that can be obtained independent of fluidic flow pattern. Based on their application in macroscopic bench-top procedures, the magnetic particles are often employed in lab-on-a-chip systems to capture biomolecules or cells of interest and transport them to a chosen point, e.g. for detection or further processing [4-6]. In most cases, the actuation is achieved by attracting the magnetic particles towards a wire or a microcoil, where they serve as magnetic labels that can be detected via magnetoresistive effects [7-9]. Further methods of choice are the optical detection of fluorescently labeled particles or the observation via a microscope $[3,6]$. The advantage of optical detection in these cases is the absence of interaction between actuation and detection, which permits an increased sensitivity of the system. Both magnetoresistive and optical

\footnotetext{
* Corresponding author. Tel.: +41 2169 36759; fax: +41 216935950.

E-mail address: ulrike.lehmann@epfl.ch (U. Lehmann).
}

approaches have been shown to allow the detection of single magnetic particles, even though differentiation between particle sizes has not yet been demonstrated $[3,10,11]$.

In this paper we present a CMOS-based system that combines guided magnetic actuation of single magnetic particles with integrated optical detection. The magnetic manipulation of the microparticles is achieved by modulating the topology of a magnetic field over the chip surface via an array of micro-coils $[12,13]$. This method allows a very precise control of the microparticle position within a glass micro-capillary and does not require external moving parts. The scale of the system permits the manipulation of single magnetic particles of different sizes $(1-30 \mu \mathrm{m})$. The use of single photon avalanche diodes (SPADs), positioned in the centers of the actuation coils ensures the optical detection with the necessary high sensitivity [14]. We evaluate the optical signal based on transit photometry, a method rather known from astronomy [15], but, as we show, also applicable for the microworld.

\section{Design and working principle}

In order to achieve a high flexibility of the system, we chose a hybrid approach, combining a CMOS chip with an exchangeable microfluidic system. The CMOS chip, comprising four 

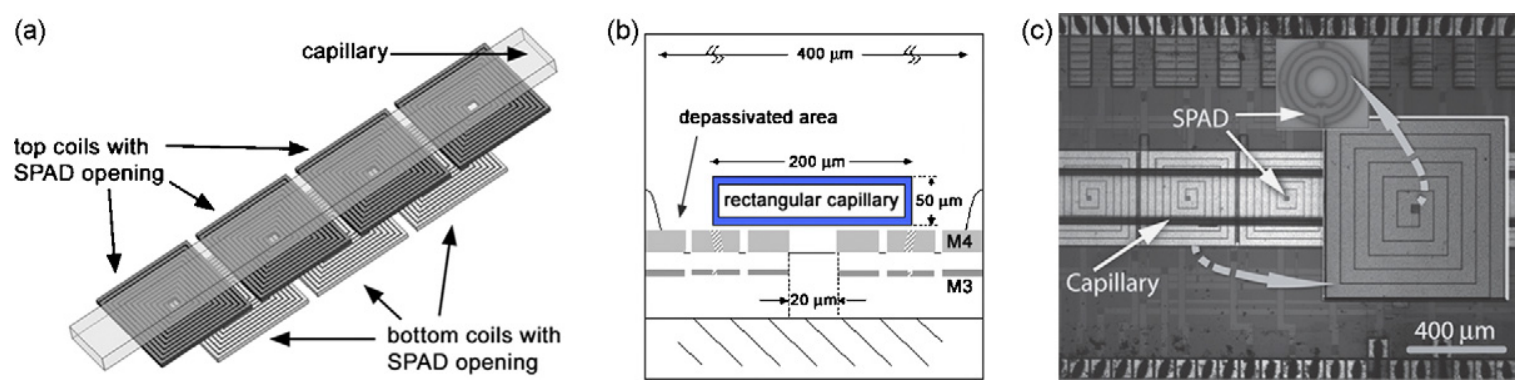

Fig. 1. Views of the CMOS chip: (a) explosion view showing the layers of the actuation system; (b) schematic sideview; (c) photograph of the system with insets of the enlarged coil and SPAD.

metal layers, contains both the actuation and detection units. The magnetic actuation is achieved via overlapping square coils with each six windings of $35 \mu \mathrm{m}$ width, located in the upper two metal layers of the chip. The SPADs, which serve as detection units, are connected to the two bottom metal layers and are positioned within a square opening of $20 \mu \mathrm{m} \times 20 \mu \mathrm{m}$ at the center of each coil. The coils are fabricated in $0.35 \mu \mathrm{m}$ 2P4M CMOS technology and the SPAD implementation is based on a design presented recently by Niclass et al. [16]. Fig. 1 presents schematic views of the system in combination with a photomicrograph of the chip including close-ups of a coil and a center SPAD. A glass micro-capillary (Vitrocom), which serves as model for future microfluidic systems, is placed on top of the coils and contains the magnetic particles suspended in an aqueous solution. The actuation of the magnetic particles is achieved via a three-phase current scheme [13]. If one coil is set to attractive mode, the adjacent coils are switched both to repulsive mode, thus increasing the magnetic field gradient, which exerts a force on the particles into the desired direction. In order to achieve attractive and repulsive modes, we alternate the direction of the coil currents. The absolute value of the dc current in an active coil is $40 \mathrm{~m}$, A which amounts to a power consumption of $9 \mathrm{~mW}$ for each coil. Fig. 2 illustrates the topology of the magnetic field during current switching and the subsequent behavior of a cloud of magnetic particles. The particles are additionally magnetized via a perpendicular homogeneous (a)
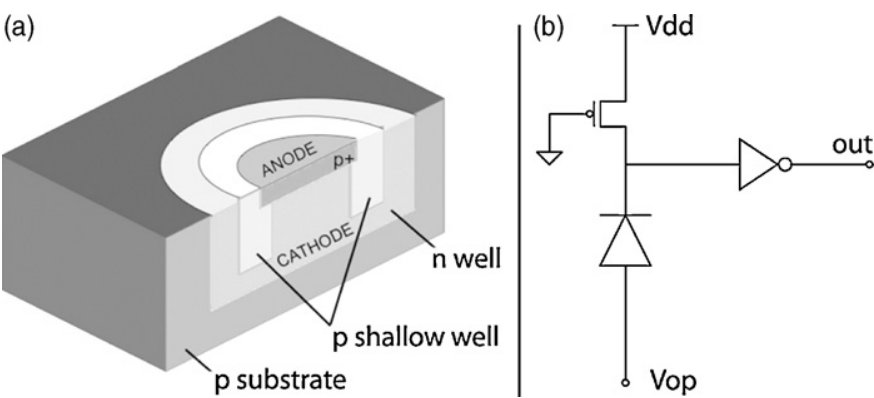

Fig. 3. Schematics of a passively quenched SPAD: (a) 3D view of the CMOS structure and (b) schematic diagram of the SPAD circuit.

magnetic field applied from outside the chip by means of an electromagnet.

\subsection{Optical detection}

For the optical detection we profit from the advantageous properties of a SPAD, which is a $\mathrm{p}-\mathrm{n}$ junction reverse biased above breakdown by an excess bias voltage $V_{\mathrm{e}}$. This configuration, schematically shown in Fig. 3, causes the optical gain to become virtually infinite, thus making single photon detection possible. When a photon is absorbed in the multiplication region, an avalanche is triggered. Thanks to a ballast resistance placed in series to the $\mathrm{p}-\mathrm{n}$ junction, the reverse bias temporarily
(ต)

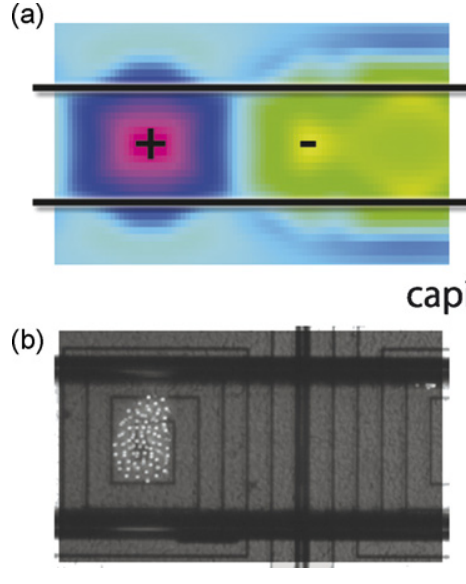

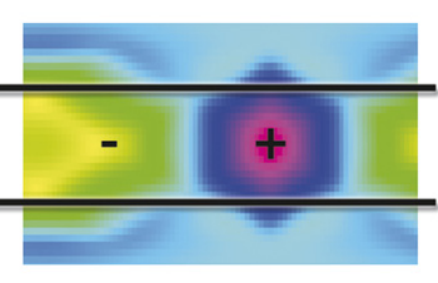

illary
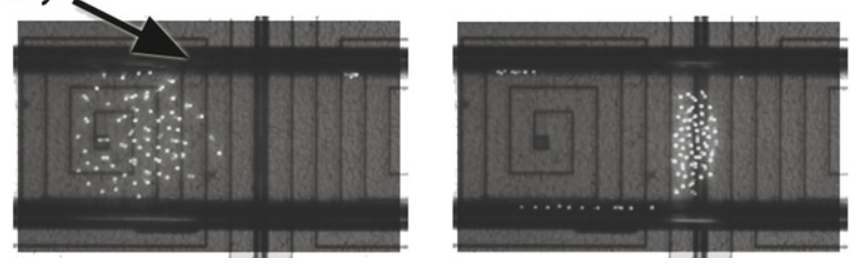

$3.5 \mathrm{mT}$

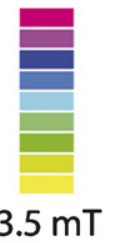

$-3.5 \mathrm{mT}$

Fig. 2. Illustration of the particle transport: (a) analytical simulation of the perpendicular field $((+)$ attractive mode, $(-)$ repulsive mode) and (b) photographs of a particle transport sequence. The images of the fluorescent magnetic particles $(\emptyset 5 \mu \mathrm{m})$ are overlaid with a photograph of the CMOS chip. The duration of the three-image sequence is $20 \mathrm{~s}$.

Please cite this article in press as: U. Lehmann, et al., Microparticle photometry in a CMOS microsystem combining magnetic actuation and in situ optical detection, Sens. Actuators B: Chem. (2007), doi:10.1016/j.snb.2007.10.021 


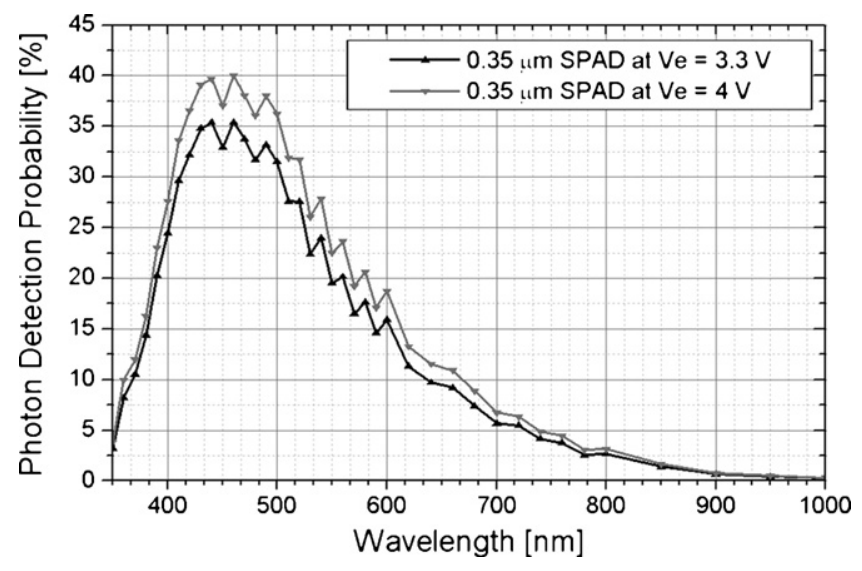

Fig. 4. Photon detection probability for different excess bias voltages $V_{\mathrm{e}}$ as a function of the wavelength of impinging photons.

drops below breakdown and quenches the avalanche. The combined duration of avalanche quenching and recharge time determines the sensor's dead time and in consequence the detection cycle of the SPAD. In this design, avalanche quenching is achieved through a passive method. The measured dead time of $40 \mathrm{~ns}$, resulting in a detection saturation count rate of $25 \mathrm{MHz}$, is well below the time a particle requires for its transit between coil centers. The voltage pulse obtained during a detection cycle is regenerated and converted to a digital pulse by an inverter. The probability that a digital pulse is generated upon impinging photons, the photon detection probability (PDP), is plotted in Fig. 4 as a function of excess bias voltage $V_{\mathrm{e}}$ and wavelength. The noise caused by a SPAD determines the minimum detectable photon flux and is characterized by the frequency of spurious pulses. These pulses are created by carriers that are not photo-generated and their effect is known as the dark count rate (DCR). It is caused by thermal or tunneling generated carriers and is therefore a strong function of temperature and excess bias voltage. In the present design, the DCR amounts to $750 \mathrm{~Hz}$ for an excess bias voltage of $3.3 \mathrm{~V}$ at room temperature. The signals of the SPADs on the chip are recorded continually via a computer interface, allowing simultaneous transport and detection.

\subsubsection{Particle photometry}

The optical detection of the actuated opaque spherical magnetic particles is based on a photometry method [17]. The SPAD is permanently illuminated using the light of a standard reflective microscope (Zeiss Axioimager). When a particle is passing over a coil center and the corresponding SPAD the number of arriving photons and in consequence the intensity of the light at the sensor is lowered, as schematically shown in Fig. 5. The transit of microparticles can thus be described as a micro-eclipse of the lightspot illuminating the SPAD. The decrease $(\Delta I)$ in measured light intensity $(I)$ depends on the radius $r$ of the passing particle (see schematic in Fig. 5c) and can be derived from the geometric constellation of the system [17]:

$$
\begin{aligned}
\frac{\Delta I}{I_{0}} & =\left(\frac{R_{\mathrm{p}}}{R_{*}}\right)^{2} \quad \text { with } R_{\mathrm{p}}=\frac{r}{\text { dist }_{\mathrm{SPAD}}} f+r \text { and } \\
R_{*} & =\left(f+\text { distSPAD }_{\mathrm{San}}(\alpha)\right.
\end{aligned}
$$

Further parameters are the properties of the microscope objective, such as its focal length $f$ and numerical aperture $\mathrm{NA}=n \sin (\alpha)$ with a refractive index of $n=1$ for air, as well as the distance between the particle and the SPAD (distSPAD), with distsPAD $\ll f$. The influence of the capillary wall $(n=1.5)$ and the liquid medium $(n=1.3)$ can be neglected since their thickness is much smaller than the distance between the light source and the particle. With all parameters known, the variation in the photon count can be converted into the particle radius, following Eq. (1).

\subsection{Particle velocity measurements}

In addition to the determination of the particle size, the presence of multiple SPADs on the chip permits the determination of further particle parameters such as the magnetization or the magnetophoretic mobility [18]. Both can be determined via the (a)

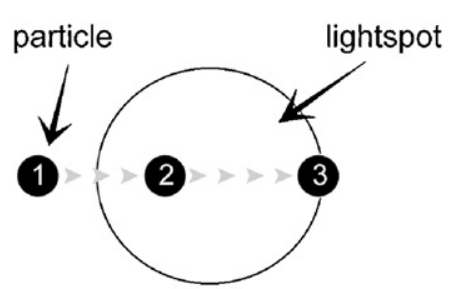

(b) intensity

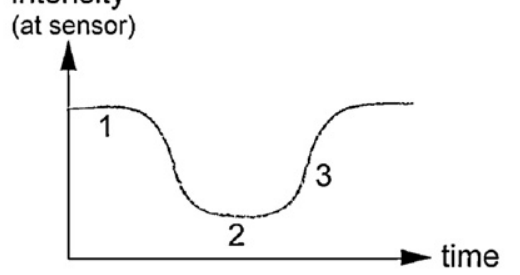

(c)

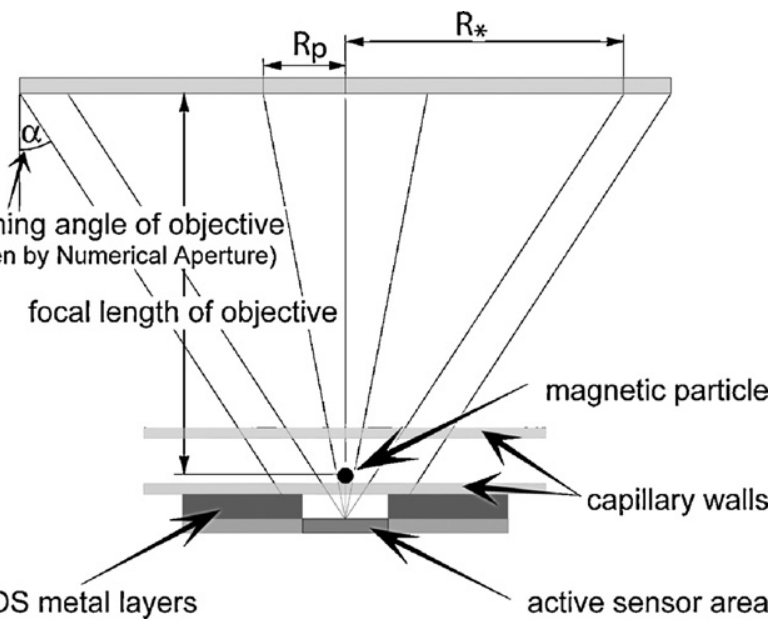

Fig. 5. Schematics of the optical detection principle based on photometry: (a) transition of the particle as seen from the sensor; (b) schematic graph of the light intensity at the sensor; (c) model of the illumination during particle transit. The back-projected particle radius $R_{\mathrm{p}}$ is proportional to the amount of light blocked. Focal length and numerical aperture are determined by the chosen objective. 
measurement of the particle speed. Observing the SPADs of two adjacent coils we can detect the moment of particle departure and subsequent arrival at the next position. With the known distance between the coil centers and the elapsed time, the particle velocity $v$ can be estimated. Since the small dimensions of the system lead to a Reynolds number smaller than 0.1, Stokes, Law can be used to calculate the viscous drag acting on the moving particles.

$F_{\mathrm{d}}=3 \pi \eta D_{\mathrm{m}} v$

$D_{\mathrm{m}}$ is the particle diameter, $v$ the particle velocity and $\eta$ is the viscosity of the surrounding medium.

Analytical field simulations indicate a fairly constant gradient of the magnetic flux density $\nabla B=8 \mathrm{~T} / \mathrm{m}$, which results in an equally constant magnetic force $F_{\mathrm{m}}$ on the particle of volume $V_{\mathrm{m}}$ due to

$F_{\mathrm{m}}=V_{\mathrm{m}} H \chi_{\mathrm{m}} \nabla B$

with the magnetic field $H$ and the particle's magnetic susceptibility $\chi_{\mathrm{m}}$.

Due to the magnetic design of the system, the magnetic particles, which are statically magnetized via an external magnetic field, are slightly lifted from the channel bottom when the nearest coil changes into the repulsive mode. Thus the particles float during the transport and are not hindered by the friction with the capillary walls. This makes the magnetic force and the viscous drag the governing forces in our system and allows us to combine Eqs. (2) and (3) in order to obtain an expression for the volume magnetization $M$ of the particle:

$M=\chi_{\mathrm{m}} H=\frac{3 \pi \eta D_{\mathrm{m}} v}{V_{\mathrm{m}} \nabla B}$

The knowledge of the magnetization $M$ enables us to determine the particles' volume magnetic moment $m$ via the known particle size and the amount of particles per gram given by the supplier.

\section{Experimental}

We examined the magnetic and optical performance of the system by manipulating magnetic particles of different diameters $(1,3,5$, and $30 \mu \mathrm{m})$ within a glass micro-capillary. The walls of the glass capillary have a thickness of $25-100 \mu \mathrm{m}$, which lowers but also smoothens the magnetic field acting on the particles with increasing thickness. The magnetic particles follow the field gradient to the next corresponding coil center, where they are optically detected by a SPAD. All experiments were performed using a standard microscope with incident illumination (Zeiss Axio Imager.A1m with Hal 100 light-source) set to an intensity that generates a photon count of $8 \mathrm{MHz}$ at the SPAD in the absence of magnetic particles. For the experiments a highly diluted solution of magnetic particles (BangsLabs, MicroMod, Spherotech) was used, which permits to manipulate single particles. Fig. 6 shows a magnetic microparticle of $1 \mu \mathrm{m}$ diameter positioned and held over a SPAD by the magnetic field.

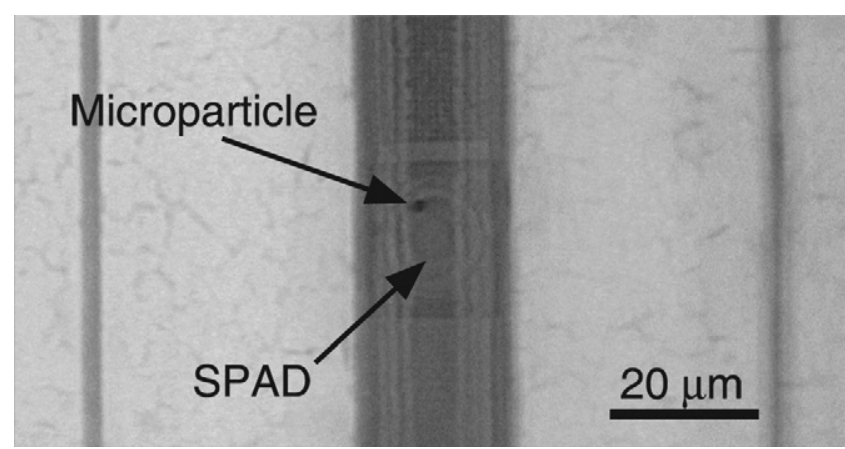

Fig. 6. Photograph of a single $1 \mu \mathrm{m}$ magnetic microparticle partially occluding a SPAD $(50 \times$ objective $)$.

\subsection{Size detection via photometry}

The experiments for the particle size detection were performed using a microscope objective with a long focal length (Zeiss "Epiplan" $10 \times, \mathrm{NA}=0.2, f=18.4 \mathrm{~mm}$ ). The SPAD, whose signal is recorded, is placed in the center of the objective's lightspot. We count the pulses emitted by the SPAD circuit using a LabView controlled computer interface (NI TB-2715, National Instruments). The signals, measured during the passage of microparticles of different sizes $(1,3$ and $5 \mu \mathrm{m})$, are plotted in Fig. 7a. Since the maximum photon count varies between experiments, the signal was normalized to permit a better comparability of the measurements. A first comparison with the theoretical model in Fig. 5b shows a strong resemblance between the curves, which indicates the validity of the photometry approach.

\subsection{Particle velocity measurements}

In parallel with the particle size measurements we examined the duration of particle displacement. The parallel observation of two adjacent SPADs permits the detection of particle departure and arrival as visible in Fig. 7b. During the time interval $\Delta t$ between the micro-eclipses over the neighboring SPADs, the magnetic particle travels the distance of six windings, which amounts to $d=210 \mu \mathrm{m}$. The ratio of the traveled distance $d$ and the elapsed time $\Delta t$ is the average velocity of the magnetic particle. Table 1 presents the average velocities measured for different types of commercially available particles used in the experiments.

\section{Results and discussion}

\subsection{Particle size determination via photometry}

An examination of Fig. 7a shows a dependence between the decrease in photon count and the particle size, as expected from Eq. (1). When comparing our measurements with the photometry model, we need to take the different wall thicknesses of the capillaries used during experiments into account, and hence the distance to the SPAD. The experiments for particles with a diameter smaller than $15 \mu \mathrm{m}$ were performed in a capillary with a wall thickness of $25 \mu \mathrm{m}$, whereas larger particles were manipulated in a capillary with $100 \mu \mathrm{m}$ thick walls. 

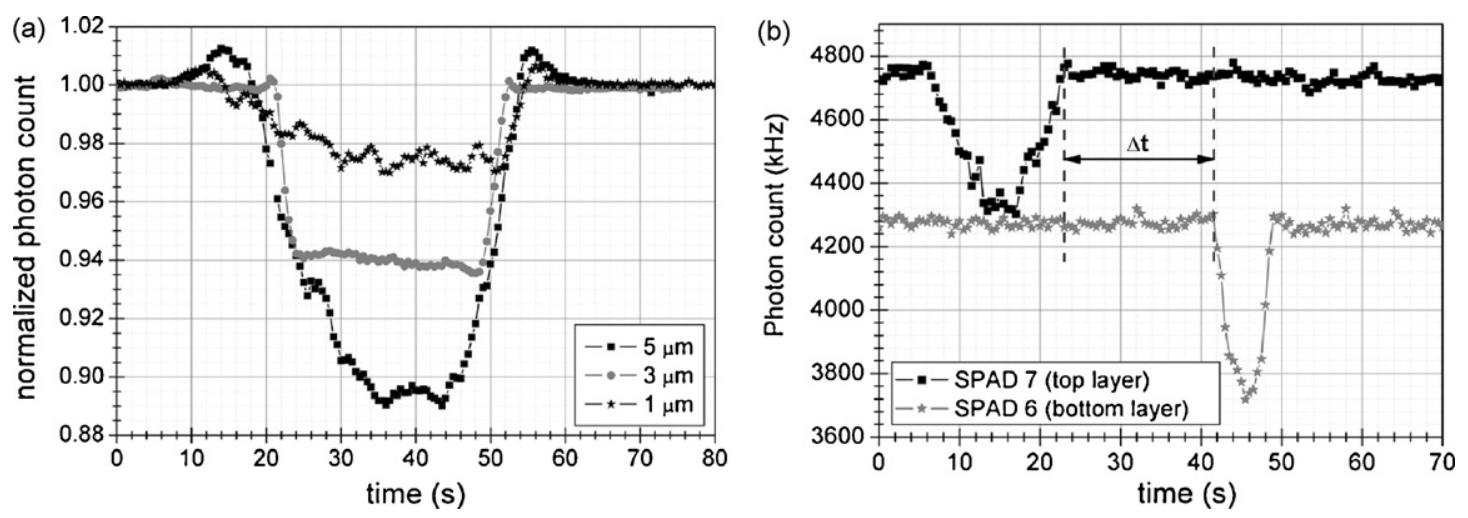

Fig. 7. Signal of a SPAD during the passage of magnetic microparticles: (a) normalized photon count of a SPAD during the transit of single particles with 1,3 and $5 \mu \mathrm{m}$ diameter and (b) photon count of two adjacent SPADs during particle transport for a particle of $5 \mu \mathrm{m}$ diameter showing the subsequent intensity drops.

Table 1

Comparison of the volume magnetization $M$ for different types of magnetic particles obtained from velocity measurements

\begin{tabular}{lllccc}
\hline & A & B & C & D \\
\hline$\emptyset(\mu \mathrm{m})$ & 1 & 1.6 & 3 & 5 & 5 \\
Type & & & $\begin{array}{c}\text { Superparamagnetic } \\
\text { BangsLabs }\end{array}$ & Ferrimagnetic \\
Vendor & & & COMPEL & COMPEL \\
Brand & 63.4 & 42.5 & 12.5 & 5.5 & 13 \\
Magnetite content (wt $\%)$ & 1.6 & 2.9 & 4.7 & 810 & 4.3 \\
$v(\mu \mathrm{m} / \mathrm{s})($ average $)$ & 3600 & 2550 & 1180 & 390 \\
$M(\mathrm{~A} / \mathrm{m})$ & & & & \\
\hline
\end{tabular}

Fig. 8 presents the theoretical and measured photon counts, demonstrating a very good accordance between the photometry model and experimental values. The increased variation for smaller particles, that is visible in the graph, can be ascribed to the size distribution of the particles, which is increasing for smaller particles, and an uncertainty in the manipulation of real particle singlets. Due to the optical observation system's constellation, the distinction between singlets and multiplets of particles was not always possible for very small particles. A comparison of the curves in Fig. 8 also shows that a decrease in capillary sidewall thickness increases the system's sensitivity towards particle size. Thus a thinner capillary sidewall will

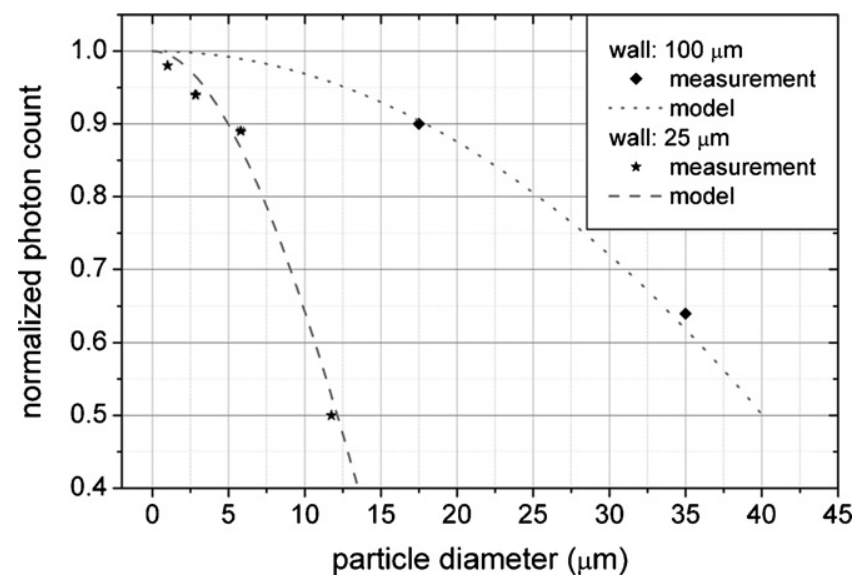

Fig. 8. Comparison of the measured and expected values for different particle diameters and varying capillary wall thicknesses. permit a better distinction between small particles. On the other hand does the decrease in distance between the sensor and the particle limit the maximum detectable size. Once the projected particle radius $R_{\mathrm{p}}$ surpasses $R *$, the photon count will drop to a minimum value common for all larger particles. Thus the sidewall width of the capillary needs to be chosen according to the range of particle sizes of interest in combination with Eq. (1).

\subsection{Particle velocity evaluation}

Our measurements of the particle speed (Table 1) show that the velocity, which is in the range of $\mu \mathrm{m} / \mathrm{s}$, increases with particle size and magnetite content. Based on the known particle size and the parameters of the system, such as viscosity and magnetic field gradient, we are able to convert the measured velocity into the magnetization $M$ according to Eq. (4). A summary of the different magnetic particles is shown in Table 1. A comparison with the magnetic content given by the particle supplier, showed a dependence between the volume magnetization and the magnetite content, which is in agreement with theory [19]. In Table 1 we can see, that a loss in magnetic force, which scales by the order of three, can be offset by an increase in magnetite content. The resulting larger magnetic moment enables smaller particles to still overcome the viscous drag force and move into a given direction. Our results additionally indicate the influence of the magnetic particle properties. Since ferromagnetic material is less easily magnetized than the superparamagnetic particles, the volume magnetization is smaller at the field strength present in the capillary. 
The particle velocity also allows an estimation of the forces obtained by the magnetic actuation. With a viscosity for water of $\eta=1 \mathrm{mPa}$ s we calculate forces on the particles in the upper $\mathrm{fN}$ range, using Eq. (2).

\section{Conclusion}

Our experiments demonstrate the advantages and the potential of the combination of magnetic actuation and optical detection in one single CMOS chip. We are able to show the possibility to actuate and detect single particles of $1-30 \mu \mathrm{m}$ diameter, with a minimum distance to the sensor of $25 \mu \mathrm{m}$, using a modular system with an exchangeable microfluidic component. We demonstrated that a decrease in the distance between the sensor and the particle can increase the particle size resolution, but limits the range of detection. The simultaneous and automated detection of velocity and size of single particles enables a wide range of particle characterizations and offers many applications in bio-analytical systems [20]. Since the principles of actuation and detection are not coupled, we can envisage using the particles as mobile substrates to transport biomaterials of interest, e.g. cells, and to perform on-chip analysis without interference between actuation and detection. With magnetic particles enclosed within or attached to a single cell, the latter can be transported and studied automatically within the system. The size can be obtained via the micro-eclipse measurements, while the determination of the velocity permits calculating the content of bound or incorporated magnetic material in the cell or its surface, linked to chosen cells properties [21].

In conclusion we can state that the combination of magnetic actuation and integrated optical detection represents a promising approach to miniaturized systems for on-chip bio-analysis.

\section{Acknowledgements}

The authors are grateful to Martin Lanz and Giovanni Nicoletti for their technical support. The research was partially supported by a grant of the Swiss National Science Foundation (Grant 200020-107372) and the Competence Centre for Materials Science and Technology (CCMX).

\section{References}

[1] M.A.M. Gijs, Magnetic bead handling on-chip: new opportunities for analytical applications, Microfluidics Nanofluidics 1 (1) (2004) 22-40.

[2] T. Deng, G.M. Whitesides, M. Radhakrishnan, Manipulation of magnetic microbeads in suspension using micromagnetic systems fabricated with soft lithography, Appl. Phys. Lett. 78 (12) (2001) 1775-1777.

[3] R. Wirix-Speetjens, W. Fyen, K.D. Xu, J. De Boeck, G. Borghs, A force study of on-chip magnetic particle transport based on tapered conductors, IEEE Trans. Magn. 41 (10) (2005) 4128-4133.

[4] U. Lehmann, C. Vandevyver, V.K. Parashar, M.A.M. Gijs, Droplet-based DNA purification in a magnetic lab-on-a-chip, Angew. Chem. Int. Edit. 45 (19) (2006) 3062-3067.

[5] J.W. Choi, C.H. Ahn, S. Bhansali, H.T. Henderson, A new magnetic bead-based, filterless bio-separator with planar electromagnet surfaces for integrated bio-detection systems, Sens. Actuator B 68 (2000) 34-39.
[6] K. Smistrup, B.G. Kjeldsen, J.L. Reimers, M. Dufva, J. Petersen, M.F. Hansen, On-chip magnetic bead microarray using hydrodynamic focusing in a passive magnetic separator, Lab Chip 5 (11) (2005) 1315-1319.

[7] D.L. Graham, H.A. Ferreira, N. Feliciano, P.P. Freitas, L.A. Clarke, M.D. Amaral, Magnetic field-assisted DNA hybridisation and simultaneous detection using micron-sized spin-valve sensors and magnetic nanoparticles, Sens. Actuator B: Chem. 107 (2) (2005) 936-944.

[8] J.C. Rife, M.M. Miller, Design and performance of GMR sensors for the detection of magnetic microbeads in biosensors, Sens. Actuator A 107 (2003) 209-218.

[9] H.A. Ferreira, N. Feliciano, D.L. Graham, P.P. Freitas, Effect of spin-valve sensor magnetostatic fields on nanobead detection for biochip applications, J. Appl. Phys. 97 (10) (2005).

[10] R. Wirix-Speetjens, W. Fyen, J. De Boeck, G. Borghs, Single magnetic particle detection: experimental verification of simulated behavior, J. Appl. Phys. 99 (10) (2006)

[11] H. Lee, A.M. Purdon, R.M. Westervelt, Micromanipulation of biological systems with microelectromagnets, IEEE Trans. Magn. 40 (4) (2004) 2991-2993.

[12] H. Lee, Y. Liu, D. Ham, R.M. Werstervelt, Integrated cell manipulation system-CMOS/microfluidic hybrid, Lab Chip 7 (2007) 331-337.

[13] A. Rida, V. Fernandez, M.A.M. Gijs, Long-range transport of magnetic microbeads using simple planar coils placed in a uniform magnetostatic field, Appl. Phys. Lett. 83 (12) (2003) 2396.

[14] A. Rochas, M. Gani, B. Furrer, P.A. Besse, R.S. Popovic, G. Ribordy, N. Gisin, Single photon detector fabricated in a complementary metal-oxidesemiconductor high-voltage technology, Rev. Sci. Instrum. 74 (7) (2003) 3263-3270

[15] Z. Sandor, Estimations of orbital parameters of exoplanets from transit photometry by using dynamical constraints, Celes. Mech. Dyn. Astron. 95 (1-4) (2006) 273-285.

[16] C. Niclass, M. Sergio, E. Charbon, A single photon avalanche diode array fabricated in $0.35-\mu \mathrm{m}$ CMOS and based on an event-driven readout for TCSPC experiments, in: Proceedings of the SPIE on Advanced Photon Counting Techniques, vol. 6372, 2006, p. 63720S.

[17] P. Sartoretti, J. Schneider, On the detection of satellites of extrasolar planets with the method of transits, Astron. Astrophys. Suppl. Ser. 134 (3) (1999) 553-560.

[18] K.E. McCloskey, J.J. Chalmers, Magnetic cell separation: characterization of magnetophoretic mobility, Anal. Chem. 75 (2003) 6868-6874.

[19] L.C. Costa, M. Valente, M.A. Sa, F. Henry, Electrical and magnetic properties of polystyrene doped with iron nanoparticles, Polym. Bull. 57 (6) (2006) 881-887.

[20] H.G. Chun, T.D. Chung, H.C. Kim, Cytometry and velocimetry on a microfluidic chip using polyelectrolytic salt bridges, Anal. Chem. 77 (8) (2005) 2490-2495.

[21] J.H. Clement, M. Schwalbe, N. Buske, K. Wagner, M. Schnabelrauch, P. Gornert, K.O. Kliche, K. Pachmann, W. Weitschies, K. Hoffken, Differential interaction of magnetic nanoparticles with tumor cells and peripheral blood cells, J. Cancer Res. Clin. Oncol. 132 (5) (2006) 287-292.

\section{Biographies}

Ulrike Lehmann was born in Rostock, Germany in 1978. She obtained her Dipl-Ing. degree in electrical engineering, with a specialization on MEMS, in 2003, at the Chemnitz Technical University, Chemnitz, Germany. She currently is a PhD student at the Ecole Polytechnique Fédérale de Lausanne (EPFL) in the Laboratory for Microsystems, where she is working on the manipulation of magnetic microparticles in liquid phases for application in biomedical systems.

Maximilian Sergio received the Dr. Eng. degree in microelectronics at the University of Bologna, Italy, in 1998. Since 2000, he has been working as full-time consultant for the Central R\&D Division of ST Microelectronics in Agrate Brianza (Milan, Italy) studying the utilization of STM CMOS technologies and CAD platforms for the development and physical implementation of innovative digital and mixed signals projects in particular in the field of smart sensors. In 2003, he received the $\mathrm{PhD}$ degree from the University of Bologna with the thesis "Design of CMOS only smart pressure sensors". From 2002 to 2004 he joined 
the "Advanced Research Center on Electronic Systems E. De Castro (ARCES)", Bologna, Italy. The focus of his activity was on design of smart sensor using standard CMOS technology. From 2005 to 2006 he was with the AQUA group at EPFL where he worked on high-performance imaging and quantum integrated circuits. Since 2007 he joined the Silicon Biosystems where he is the responsible for analog and mixed-signal design of integrated sensors and actuators for living cell manipulation.

Stefano Pietrocola was born in Torino, Italy, in 1982. He received the BS degree in engineering mathematics from Politecnico di Torino in 2005, and the MS degree in micro and nanotechnologies for integrated systems in collaboration with the Politecnico di Torino, the INP de Grenoble and the EPFL, in September 2006. He is currently working at a spin-off of the University of Turin, Nanovector, on the manipulation of nanoparticles for drug delivery systems.

Emile Dupont was born in Aosta, Italy, in 1982. He received his Bachelor degree in information engineering from Politecnico di Torino in 2004 and his Master degree in 2006 in the joint program held by Politecnico di Torino, INP Grenoble and EPF Lausanne in micro and nanotechnologies with a master thesis titled "From Macro to Micro Computational Fluid Dynamics: CFD Simulations at CERN". He is currently pursuing his PhD at EPFL in the Microsystems' Group leaded by Prof. Gijs, working on the manipulation of paramagnetic beads for Bio-MEMS applications.

Cristiano Niclass received the MSc degree in microtechnology with emphasis in applied photonics from EPFL in 2003. In May 2003, he joined the Quantum Architecture Group (AQUA), where he is working toward the PhD degree. His interests include high-speed and low-noise digital and mixed-mode applicationspecific integrated circuits with emphasis on high-performance imaging. $\mathrm{He}$ is currently in charge of the design, implementation, and evaluation of fully integrated two- and three-dimensional CMOS image sensors based on single photon avalanche diodes. He is also involved in the design of time discrimination devices with picosecond resolution implemented in conventional technologies. Mr. Niclass is a member of the Institute of Electrical and Electronics Engineers (IEEE).
Martin A.M. Gijs received his degree in physics in 1981 from the Katholieke Universiteit Leuven, Belgium, and his PhD degree in physics at the same university in 1986. He joined the Philips Research Laboratories in Eindhoven, The Netherlands, in 1987. Subsequently, he has worked there on microand nano-fabrication processes of high critical temperature superconducting Josephson and tunnel junctions, the micro-fabrication of microstructures in magnetic multilayers showing the giant magnetoresistance effect, the design and realisation of miniaturised motors for hard disk applications and the design and realisation of planar transformers for miniaturised power applications. He joined the Swiss Federal Institute of Technology Lausanne (Ecole Polytechnique Fédérale de Lausanne) in 1997. He presently is a professor in the Institute of Micro-electronics and Microsystems, where he is responsible for the Microsystems Technology Group. His main interests are in developing technologies for novel magnetic devices, new microfabrication technologies for microsystems fabrication in general and the development and use of microsystems technologies for biomedical applications in particular.

Edoardo Charbon received the Diploma from ETH-Zürich, the MS from UCSD, and the PhD from UC-Berkeley, all in EECS, in 1988, 1991, and 1995, respectively. From 1995 to 2000, he was with Cadence Design Systems, where he was the architect of the company's initiative for intellectual property protection. In 2000, he joined Canesta Inc. as its Chief Architect, leading the development of wireless 3D CMOS image sensors. Since November 2002, he has been a member of the Faculty of EPFL, where he has founded the Quantum Architecture Group (AQUA), a research group devoted to the field of high-accuracy imaging and ultra low-power wireless embedded systems. $\mathrm{He}$ has consulted for numerous organizations, including Texas Instruments, Hewlett-Packard, Cadence, and the Carlyle Group. He has authored or coauthored over 90 articles in technical journals and conference proceedings and two books, and he holds nine patents. His research interests include 3D microimaging, bio-imaging, integrated optical communications, intellectual property protection, substrate modeling and characterization, and micromachined sensor design. 